\title{
Higher accuracy analytical approximations to a nonlinear oscillator with discontinuity by He's homotopy perturbation method
}

A. Beléndez, A. Hernández, T. Beléndez, C. Neipp and A. Márquez

Departamento de Física, Ingeniería de Sistemas y Teoría de la Señal. Universidad de Alicante. Apartado 99. E-03080 Alicante. SPAIN

E-mail: a.belendez@ua.es

Corresponding author: A. Beléndez

Phone: +34-96-5903651

Fax: +34-96-5909750 


\begin{abstract}
He's homotopy perturbation method is used to calculate higher-order approximate periodic solutions of a nonlinear oscillator with discontinuity for which the elastic force term is proportional to $\operatorname{sgn}(x)$. We find He's homotopy perturbation method works very well for the whole range of initial amplitudes, and the excellent agreement of the approximate frequencies and periodic solutions with the exact ones has been demonstrated and discussed. Only one iteration leads to high accuracy of the solutions with a maximal relative error for the approximate period of less than $1.56 \%$ for all values of oscillation amplitude, while this relative error is $0.30 \%$ for the second iteration and as low as $0.057 \%$ when the third-order approximation is considered. Comparison of the result obtained using this method with those obtained by different harmonic balance methods reveals that He's homotopy perturbation method is very effective and convenient.
\end{abstract}

Keywords: Nonlinear oscillator; Approximate solutions; Homotopy perturbation method. 


\section{Introduction}

The study of nonlinear problems is of crucial importance not only in all areas of physics but also in engineering and other disciplines, since most phenomena in our world are essentially nonlinear and are described by nonlinear equations. It is very difficult to solve nonlinear problems and, in general, it is often more difficult to get an analytic approximation than a numerical one for a given nonlinear problem. There are several methods used to find approximate solutions to nonlinear problems, such as perturbation techniques [1-6], harmonic balance based methods [6-10] or other techniques [11-17]. An excellent review on some asymptotic methods for strongly nonlinear equations can be found in detail in references [18] and [19].

In the present paper we obtain higher-order analytical approximations to the periodic solutions to a nonlinear oscillator with discontinuity for which the elastic restoring force is an antisymmetric and constant force. To do this, we apply He's homotopy perturbation method [20-38]. This type of oscillator has been analyzed by Liu [39] and by Özis and Yildirim [34] applying the first-order homotopy perturbation method. They also make the comparison of two distinct adaptations of the first-order homotopy perturbation method for determining frequency-amplitude relation of the nonlinear oscillator with discontinuities. However, higher-order analytical approximate solutions have not been obtained for this oscillator using He's homotopy perturbation method. As we can see, the results presented in this paper reveal that the method is very effective and convenient for conservative nonlinear oscillators for which the restoring force has a non-polynomial form.

\section{Solution procedure}

This paper considers the following nonlinear oscillator with discontinuity

$$
\frac{\mathrm{d}^{2} x}{\mathrm{~d} t^{2}}+\operatorname{sgn}(x)=0
$$

with initial conditions

$$
x(0)=A \quad \text { and } \quad \frac{\mathrm{d} x}{\mathrm{~d} t}(0)=0
$$


and $\operatorname{sgn}(x)$ is defined by

$$
\operatorname{sgn}(x)= \begin{cases}-1, & x<0 \\ +1, & x \geq 0\end{cases}
$$

All the solutions to Eq. (1) are periodic. We denote the angular frequency of these oscillations by $\omega$ and note that one of our major tasks is to determine $\omega(A)$, i.e., the functional behaviour of $\omega$ as a function of the initial amplitude $A$.

There is no small parameter in Eq. (1), so the traditional perturbation methods can not be applied directly, moreover the equation involves discontinuity. Due to the fact that the homotopy perturbation method requires neither a small parameter nor a linear term in a differential equation, we can approximately solve Eq. (1) using the homotopy perturbation method. In this method, an artificial perturbation equation is constructing by embedding an artificial parameter $p \in[0,1]$, which is used as expanding parameter. This technique yields a very rapid convergence of the solution series; in most cases, only one iteration leads to high accuracy of the solution. This method provides an effective and convenient mathematical tool for nonlinear differential equations [18, 19].

Equation (1) can be re-written in the form

$$
\frac{\mathrm{d}^{2} x}{\mathrm{~d} t^{2}}+x=x-\operatorname{sgn}(x)
$$

For Eq. (4) we can establish the following homotopy

$$
\frac{\mathrm{d}^{2} x}{\mathrm{~d} t^{2}}+x=p[x-\operatorname{sgn}(x)]
$$

where $p$ is the homotopy parameter. When $p=0$, Eq. (5) becomes a linear differential equation for which an exact solution can be calculated; for $p=1$, Eq. (5) then becomes the original problem. Now the homotopy parameter $p$ is used to expand the solution $x(t)$ and the square of the unknown angular frequency $\omega$ as follows

$$
x(t)=x_{0}(t)+p x_{1}(t)+p^{2} x_{2}(t)+\ldots
$$




$$
1=\omega^{2}-p \alpha_{1}-p^{2} \alpha_{2}-\ldots
$$

where $\alpha_{i}(i=1,2, \ldots)$ are to be determined.

Substituting Eqs. (6) and (7) into Eq. (5)

$$
\begin{gathered}
\left(x_{0}^{\prime \prime}+p x_{1}^{\prime \prime}+p^{2} x_{2}^{\prime \prime}+\ldots\right)+\left(\omega^{2}-p \alpha_{1}-p^{2} \alpha_{2}-\ldots\right)\left(x_{0}+p x_{1}+p^{2} x_{2}+\ldots\right)= \\
=p\left[\left(x_{0}+p x_{1}+p^{2} x_{2}+\ldots\right)-\operatorname{sgn}\left(x_{0}+p x_{1}+p^{2} x_{2}+\ldots\right)\right]
\end{gathered}
$$

and collecting the terms of the same power of $p$, we obtain a series of linear equations, of which we write only the first four

$$
\begin{array}{ll}
x_{0}^{\prime \prime}+\omega^{2} x_{0}=0, & x_{0}(0)=A, \quad x_{0}^{\prime}(0)=0 \\
x_{1}^{\prime \prime}+\omega^{2} x_{1}=\left(1+\alpha_{1}\right) x_{0}-\operatorname{sgn}\left(x_{0}\right), & x_{1}(0)=x_{1}^{\prime}(0)=0 \\
x_{2}^{\prime \prime}+\omega^{2} x_{2}=\alpha_{2} x_{0}+\left(1+\alpha_{1}\right) x_{1}, & x_{2}(0)=x_{2}^{\prime}(0)=0 \\
x_{3}^{\prime \prime}+\omega^{2} x_{3}=\alpha_{3} x_{0}+\alpha_{2} x_{1}+\left(1+\alpha_{1}\right) x_{2}, & x_{3}(0)=x_{3}^{\prime}(0)=0
\end{array}
$$

In Eqs. (9)-(12) we have taken into account the following expression

$$
\begin{array}{r}
f(x)=f\left(x_{0}+p x_{1}+p^{2} x_{2}+p^{3} x_{3}+\ldots\right)=f\left(x_{0}\right)+p\left(\frac{\mathrm{d} f(x)}{\mathrm{d} x}\right)_{x=x_{0}} x_{1}+ \\
+p^{2}\left[\left(\frac{\mathrm{d} f(x)}{\mathrm{d} x}\right)_{x=x_{0}} x_{2}+\frac{1}{2}\left(\frac{\mathrm{d}^{2} f(x)}{\mathrm{d} x^{2}}\right)_{x=x_{0}} x_{1}^{2}\right]+O\left(p^{3}\right)
\end{array}
$$

where $f(x)=\operatorname{sgn}(x)$ and

$$
\frac{\mathrm{d} \operatorname{sgn}(x)}{\mathrm{d} x}=\frac{\mathrm{d}^{2} \operatorname{sgn}(x)}{\mathrm{d} x^{2}}=\ldots=0 \text { for } x \neq 0
$$


and then

$$
\operatorname{sgn}\left(x_{0}+p x_{1}+p^{2} x_{2}+\ldots\right)=\operatorname{sgn}\left(x_{0}\right)
$$

The solution of Eq. (9) is

$$
x_{0}(t)=A \cos \omega t
$$

Substitution of this result into the right side of Eq. (10) gives

$$
x_{1}^{\prime \prime}+\omega^{2} x_{1}=\left(1+\alpha_{1}\right) A \cos \omega t-\operatorname{sgn}(A \cos \omega t)
$$

It is possible to do the following Fourier series expansion

$$
\operatorname{sgn}(\cos \omega t)=\sum_{n=0}^{\infty} a_{2 n+1} \cos [(2 n+1) \omega t]==a_{1} \cos \omega t+a_{3} \cos 3 \omega t+\ldots
$$

where

$$
a_{2 n+1}=\frac{4}{\pi} \int_{0}^{\pi / 2} \operatorname{sgn}(A \cos \tau) \cos [(2 n+1) \tau] \mathrm{d} \tau=(-1)^{n} \frac{4}{(2 n+1) \pi}
$$

The first term of the expansion in Eq. (16) is given by

$$
a_{1}=\frac{4}{\pi} \int_{0}^{\pi / 2} \operatorname{sgn}(A \cos \tau) \cos \tau \mathrm{d} \tau=\frac{4}{\pi}
$$

Substituting Eq. (16) into Eq. (15), we have

$$
x_{1}^{\prime \prime}+\omega^{2} x_{1}=\left[\left(1+\alpha_{1}\right) A-a_{1}\right] \cos \omega t-\sum_{n=1}^{\infty} a_{2 n+1} \cos [(2 n+1) \omega t]
$$

No secular terms in $x_{1}(t)$ requires eliminating contributions proportional to $\cos \omega t$ on the right-hand side of Eq. (19) and we obtain 


$$
\alpha_{1}=-1+\frac{a_{1}}{A}=-1+\frac{4}{\pi A}
$$

From Eqs. (7), (18) and (20), writing $p=1$, we can easily find that the first-order approximate frequency is

$$
\omega_{1}(A)=\sqrt{\frac{a_{1}}{A}}=\frac{2}{\sqrt{\pi A}}=\frac{1.128379}{\sqrt{A}}
$$

and the first-order approximate period can be obtained as follows

$$
T_{1}(A)=\pi \sqrt{\pi A}=5.568328 \sqrt{A}
$$

which agrees exactly with Liu's solution obtained by He's modified Lindsted-Poincaré method [39], with Rafei et al.'s solution obtained by He's variational iteration method [40] and with $\mathrm{Wu}$ et al.'s solution obtained by the low-order harmonic balance method [41].

Now in order to obtain the correction term $x_{1}$ for the periodic solution $x_{0}$ we consider the following procedure. Taking into account Eqs. (19) and (20), we re-write Eq. (19) in the form

$$
x_{1}^{\prime \prime}+\omega^{2} x_{1}=-\sum_{n=1}^{\infty} a_{2 n+1} \cos [(2 n+1) \omega t]
$$

with initial conditions $x_{1}(0)=0$ and $x_{1}^{\prime}(0)=0$. The periodic solution to Eq. (23) can be written

$$
x_{1}(t)=\sum_{n=0}^{\infty} b_{2 n+1} \cos [(2 n+1) \omega t]
$$

Substituting Eq. (24) into Eq. (23) we can write the following expression for the coefficients $b_{2 n+1}$

$$
b_{2 n+1}=\frac{a_{2 n+1}}{4 n(n+1) \omega^{2}}=\frac{(-1)^{n}}{n(n+1)(2 n+1) \pi \omega^{2}},
$$


for $n \geq 1$. Taking into account that $x_{1}(0)=0$, Eq. (24) gives

$$
b_{1}=-\sum_{n=1}^{\infty} b_{2 n+1}=\frac{\pi-3}{\pi \omega^{2}}=\frac{\sigma}{\omega^{2}}
$$

where

$$
\sigma=1-\frac{3}{\pi}
$$

Substituting Eqs. (14), (20), (24), (25) and (26) into Eq. (11) gives the following equation for gives the following equation for $x_{2}(t)$

$$
x_{2}^{\prime \prime}+\omega^{2} x_{2}=\alpha_{2} A \cos \omega t+\frac{\sigma a_{1}}{A \omega^{2}} \cos \omega t+\sum_{n=1}^{\infty} \frac{a_{2 n+1}}{4 n(n+1) A \omega^{2}} \cos [(2 n+1) \omega t]
$$

The secular term in the solution for $x_{2}(t)$ can be eliminated if

$$
\alpha_{2}=-\frac{\sigma a_{1}}{A^{2} \omega^{2}}=\frac{12-4 \pi}{\pi^{2} A^{2} \omega^{2}}
$$

From Eqs. (7), (20) and (29), and taking $p=1$, one can easily obtain the following expression for the second-order approximate frequency is

$$
\omega_{2}(A)=\frac{1}{\sqrt{2 A}} \sqrt{a_{1}+\sqrt{a_{1}^{2}-4 \sigma a_{1}}}=\sqrt{\frac{2+2 \sqrt{4-\pi}}{\pi A}}=\frac{1.107452}{\sqrt{A}}
$$

and the second-order approximate period is given by

$$
T_{2}(A)=5.672551 \sqrt{A}
$$

With the requirement of Eq. (29), we can re-write Eq. (28) in the form 


$$
x_{2}^{\prime \prime}+\omega^{2} x_{2}=\sum_{n=1}^{\infty} \frac{a_{2 n+1}}{4 n(n+1) A \omega^{2}} \cos [(2 n+1) \omega t]
$$

with initial conditions $x_{2}(0)=0$ and $x_{2}^{\prime}(0)=0$. The solution of this equation is

$$
x_{2}(t)=\sum_{n=0}^{\infty} c_{2 n+1} \cos [(2 n+1) \omega t]
$$

Substituting Eq. (33) into Eq. (32) we obtain the following expression for the coefficients $c_{2 n+1}$

$$
c_{2 n+1}=-\frac{a_{1} a_{2 n+1}}{16 n^{2}(n+1)^{2} A \omega^{4}}=\frac{(-1)^{n+1}}{n^{2}(n+1)^{2}(2 n+1) \pi^{2} A \omega^{4}}
$$

for $n \geq 1$. Taking into account that $x_{2}(0)=0$, Eq. (33) gives

$$
c_{1}=-\sum_{n=1}^{\infty} c_{2 n+1}=\frac{\pi^{2}+24 \pi-66}{6 \pi^{2} A \omega^{4}}=\frac{\lambda}{A \omega^{4}}
$$

where

$$
\lambda=\frac{\pi^{2}+24 \pi-66}{6 \pi^{2}}
$$

Substitution of Eqs. (14), (20), (24), (25), (26), (29) and (33) into Eq. (12) gives the following equation for gives the following equation for $x_{3}(t)$

$$
\begin{aligned}
x_{3}^{\prime \prime}+\omega^{2} x_{3} & =\alpha_{3} A \cos \omega t+\frac{\lambda a_{1}}{A^{2} \omega^{4}} \cos \omega t-\frac{\sigma^{2} a_{1}}{A^{2} \omega^{4}} \cos \omega t \\
- & \sum_{n=1}^{\infty} \frac{\sigma^{2} a_{2 n+1}}{4 n(n+1) A^{2} \omega^{4}} \cos [(2 n+1) \omega t]-\sum_{n=1}^{\infty} \frac{a_{1}^{2} a_{2 n+1}}{16 n^{2}(n+1)^{2} A^{2} \omega^{4}} \cos [(2 n+1) \omega t]
\end{aligned}
$$

The secular term in the solution for $x_{3}(t)$ can be eliminated if 


$$
\alpha_{3}=\frac{\sigma^{2} a_{1}-\lambda a_{1}}{A^{3} \omega^{4}}=\frac{240-120 \pi+14 \pi^{2}}{3 \pi^{3} A^{3} \omega^{4}}
$$

From Eqs. (7), (20), (29) and (38), and taking $p=1$, one can easily obtain the following expression for the third-order approximate frequency is

$$
\omega_{3}(A)=\frac{1.111358}{\sqrt{A}}
$$

and the third-order approximate period is given by

$$
T_{3}(A)=5.653609 \sqrt{A}
$$

With the requirement of Eq. (38), we can re-write Eq. (37) in the form

$$
x_{3}^{\prime \prime}+\omega^{2} x_{3}=-\sum_{n=1}^{\infty} \frac{\sigma^{2} a_{2 n+1}}{4 n(n+1) A^{2} \omega^{4}} \cos [(2 n+1) \omega t]-\sum_{n=1}^{\infty} \frac{a_{1}^{2} a_{2 n+1}}{16 n^{2}(n+1)^{2} A^{2} \omega^{4}} \cos [(2 n+1) \omega t]
$$

with initial conditions $x_{3}(0)=0$ and $x_{3}^{\prime}(0)=0$. The solution of this equation is

$$
x_{3}(t)=\sum_{n=0}^{\infty} d_{2 n+1} \cos [(2 n+1) \omega t]
$$

Substituting Eq. (42) into Eq. (41) we obtain the following expression for the coefficients $d_{2 n+1}$

$$
d_{2 n+1}=\frac{(-1)^{n+1}[n(n+1)(\pi-3)+1]}{n^{3}(n+1)^{3}(2 n+1) \pi^{3} A^{2} \omega^{6}}
$$

for $n \geq 1$. Taking into account that $x_{3}(0)=0$, Eq. (41) gives 


$$
d_{1}=-\sum_{n=1}^{\infty} d_{2 n+1}=\frac{\pi^{3}-32 \pi^{2}+234 \pi-450}{6 \pi^{3} A^{2} \omega^{6}}
$$

\section{Comparison with the exact and other approximate solutions}

We illustrate the accuracy of the modified approach by comparing the approximate solutions previously obtained with the exact period $T_{e}$ and other results in the literature. In particular, we will consider the solution of Eq. (1) using the harmonic balance method [41]. This method is a procedure for determining analytical approximations to the periodic solutions of differential equations using a truncated Fourier series representation [6]. Like the homotopy perturbation method, the harmonic balance method can be applied to nonlinear oscillatory problems where a linear term does not exist, the nonlinear terms are not small, and there is no perturbation parameter. However, it is very difficult to use the harmonic balance method to construct higher-order analytical approximations because this method requires solving analytical solutions of sets of algebraic equations with very complex nonlinearities.

For this nonlinear problem, the exact periodic solution and the exact period are given by the following equations [41]

$$
\begin{gathered}
x_{e}(t)= \begin{cases}-\frac{t^{2}}{2}+A, & 0 \leq t \leq \frac{T_{e}}{4} \\
\frac{t^{2}}{2}-2 \sqrt{2 A} t+3 A, & \frac{T_{e}}{4}<t \leq \frac{3 T_{e}}{4}, \\
-\frac{t^{2}}{2}+4 \sqrt{2 A} t-15 A & \frac{3 T_{e}}{4}<t \leq T_{e}\end{cases} \\
T_{e}(A)=4 \sqrt{2 A}=5.656854 \sqrt{A}
\end{gathered}
$$

The period values and their relative errors $(R E)$ obtained in this paper applying He's homotopy perturbation method to this nonlinear oscillator with discontinuity are the following 


$$
\begin{array}{cc}
T_{1}(A)=5.568328 \sqrt{A} & \text { Relative error }=1.56 \% \\
T_{2}(A)=5.672551 \sqrt{A} & \text { Relative error }=0.30 \% \\
T_{3}(A)=5.653609 \sqrt{A} & \text { Relative error }=0.057 \%
\end{array}
$$

Wu, Sun and Lim [41] approximately solved Eq. (1) using an improved harmonic balance method that incorporates salient features of both Newton's method and the harmonic balance method. They achieved the following results for the first, second and third approximation orders

$$
\begin{array}{cc}
T_{W S L 1}(A)=5.568328 \sqrt{A} & \text { Relative error }=1.56 \% \\
T_{W L L 2}(A)=5.674402 \sqrt{A} & \text { Relative error }=0.31 \% \\
T_{W S L 3}(A)=5.650976 \sqrt{A} & \text { Relative error }=0.10 \%
\end{array}
$$

It is clear that at the third approximation order, the result obtained in this paper is better than those obtained previously by other authors.

An easy and direct calculation gives the following series representation for the exact solution $x_{e}(t)$ (Eq. (45))

$$
x_{e}(t)=\frac{32 A}{\pi^{3}} \sum_{n=0}^{\infty} \frac{(-1)^{n}}{(2 n+1)^{3}} \cos \left[(2 n+1) \omega_{e} t\right]
$$

where

$$
\omega_{e}(A)=\frac{2 \pi}{T_{e}(A)}=\frac{\pi}{2 \sqrt{2 A}}=\frac{1.110721}{\sqrt{A}}
$$

The first terms of the Fourier expansion in Eq. (53) are 


$$
\begin{aligned}
x_{e}(t)= & 1.03205 A \cos \left(\omega_{e} t\right)-0.038224 A \cos \left(3 \omega_{e} t\right)+0.00825639 A \cos \left(5 \omega_{e} t\right) \\
& -0.00300889 A \cos \left(7 \omega_{e} t\right)+0.00141571 A \cos \left(9 \omega_{e} t\right)-\ldots
\end{aligned}
$$

The first, the second and the third order approximate solutions obtained in this paper are

$$
\begin{aligned}
x_{a 1}(t)= & x_{0}\left(t, \omega_{1}\right)+x_{1}\left(t, \omega_{1}\right) \\
& =1.03540 A \cos \left(\omega_{1} t\right)-0.0416667 A \cos \left(3 \omega_{1} t\right)+0.00833333 A \cos \left(5 \omega_{1} t\right) \\
& -0.00297619 \cos \left(7 \omega_{1} t\right)+0.00138889 \cos \left(9 \omega_{1} t\right)-\ldots \\
x_{a 2}(t)= & x_{0}\left(t, \omega_{2}\right)+x_{1}\left(t, \omega_{2}\right)+x_{2}\left(t, \omega_{2}\right) \\
& =1.03146 A \cos \left(\omega_{2} t\right)-0.037643 A \cos \left(3 \omega_{2} t\right)+0.00827703 A \cos \left(5 \omega_{2} t\right) \\
& -0.00302291 \cos \left(7 \omega_{2} t\right)+0.00142316 \cos \left(9 \omega_{2} t\right)-\ldots \\
x_{a 3}(t)= & x_{0}\left(t, \omega_{3}\right)+x_{1}\left(t, \omega_{3}\right)+x_{2}\left(t, \omega_{3}\right)+x_{3}\left(t, \omega_{3}\right) \\
= & 1.03216 A \cos \left(\omega_{3} t\right)-0.038333 A \cos \left(3 \omega_{3} t\right)+0.00825087 A \cos \left(5 \omega_{3} t\right) \\
& -0.00300598 \cos \left(7 \omega_{3} t\right)+0.00141422 \cos \left(9 \omega_{3} t\right)-\ldots
\end{aligned}
$$

where $\omega_{1}(A), \omega_{2}(A)$ and $\omega_{3}(A)$ are given by Eqs. (21), (30) and (39) respectively. If we compare the approximate solutions for the first, the second and the third analytical approximations with the Fourier expansion of the exact solution, we can see that $x_{a 3}(t)$ is a better approximation to the exact solution. These results are an indication of the accuracy of the homotopy perturbation method as applied to this particular problem and show that it provides an excellent approximation to the solution of Eq. (1).

\section{Conclusions}

He's homotopy perturbation method has been used to obtain three approximate frequencies for a conservative antisymmetric, constant force nonlinear oscillator for which 
the elastic force term is proportional to $\operatorname{sgn}(x)$. Excellent agreement between approximate frequencies and the exact one has been demonstrated and discussed, and the discrepancy of the third-order approximate period, $T_{3}(A)$, with respect to the exact one is as low as $0.057 \%$. Furthermore, we concluded that discontinuous function had no tangible effect on the effectivity of the method. Finally, we think that the method has great potential and can be applied to other strongly nonlinear oscillators with non-polynomial terms.

\section{Acknowledgements}

This work was supported by the "Ministerio de Educación y Ciencia", Spain, under project FIS2005-05881-C02-02, and by the "Generalitat Valenciana", Spain, under project ACOMP/2007/020. 


\section{References}

[1] J. H. He, "A new perturbation technique which is also valid for large parameters", $J$. Sound Vib. 229, 1257-1263 (2000).

[2] J. H. He, "Modified Lindstedt-Poincare methods for some non-linear oscillations. Part III: double series expansion", Int. J. Non-linear Sci. Numer. Simulation 2, $317-$ $320(2001)$.

[3] T. Özis and A. Yildirim, "Determination of periodic solution for a $\mathrm{u}^{1 / 3}$ force by He's modified Lindstedt-Poincaré method", J. Sound Vib. 301, 415-419 (2007).

[4] P. Amore and F. M. Fernández, "Exact and approximate expressions for the period of anharmonic oscillators", Eur. J. Phys. 26, 589-601 (2005).

[5] P. Amore, A. Raya and F. M. Fernández, "Alternative perturbation approaches in classical mechanics”, Eur. J. Phys. 26, 1057-1063 (2005).

[6] R. E. Mickens, Oscillations in Planar Dynamics Systems (World Scientific, Singapore 1996).

[7] C. W. Lim, B. S. Wu and W. P. Sun, "Higher accuracy analytical approximations to the Duffing-harmonic oscillator", J. Sound Vib. 296, 1039-1045 (2006).

[8] A. Beléndez, A. Hernández, A. Márquez, T. Beléndez and C. Neipp, "Analytical approximations for the period of a simple pendulum”, Eur. J. Phys. 27, 539-551 (2006).

[9] A. Beléndez, A. Hernández, T. Beléndez, M.L. Álvarez, S. Gallego, M. Ortuño and C. Neipp "Application of the harmonic balance method to a nonlinear oscillator typified by a mass attached to a stretched wire", J. Sound Vib. 302, 1018-1029 (2007). 
[10] G. R. Itovich and J. L. Moiola, "On period doubling bifurcations of cycles and the harmonic balance method", Chaos, Solitons \& Fractals 27, 647-665 (2005).

[11] D. H. Shou and J. H. He, "Application of parameter-expanding method to strongly nonlinear oscillators", Int. J. Non-linear Sci. Numer. Simulation 8 (1), 121-124 (2007).

[12] J. H. He and X. H. Wu, "Construction of solitary solution and compact on-like solution by variational iteration method", Chaos, Solitons \& Fractals 29, 108-113 (2006).

[13] J. H. He, "Variational approach for nonlinear oscillators", Chaos, Solitons \& Fractals 34, 1430-1439 (2007).

[14] M. Tatari and M. Dehghan, "The use of He's variational iteration method for solving a Fokker-Planck equation”, Phys. Scr. 74, 310-316 (2006).

[15] M. Tatari and M. Dehghan, "On the convergence of He's variational iteration method", J. Comput. Appl. Math. 207, 121-128 (2007).

[16] M. Tatari and M. Dehghan, "Solution of problems in calculus of variations via He's variational iteration method", Phys. Lett. A 362, 401-406 (2007).

[17] B. Bathia, M. S. M. Noorani and I. Hashim, "Numerical simulation of the generalizaed Huxley equation by He's variational iteration method", Appl. Math. Comput. 186, 1322-1325 (2007).

[18] J. H. He, Non-perturbative methods for strongly nonlinear problems Dissertation (De-Verlag im Internet GmbH, Berlin 2006). 
[19] J. H. He, "Some asymptotic methods for strongly nonlinear equations", Int. J. Mod. Phys. B, 20, 1141-1199 (2006).

[20] J. H. He, "New interpretation of homotopy perturbation method", Int. J. Mod. Phys. $B, 20,2561-2568$ (2006).

[21] J. H. He, "The homotopy perturbation method for nonlinear oscillators with discontinuities", Appl. Math. Comp. 151, 287-292 (2004).

[22] X. C. Cai, W. Y. Wu and M. S. Li, “Approximate period solution for a kind of nonlinear oscillator by He's perturbation method", Int. J. Non-linear Sci. Numer. Simulation 7 (1), 109-117 (2006).

[23] L. Cveticanin, "Homotopy-perturbation for pure nonlinear differential equation", Chaos, Solitons \& Fractals 30, 1221-1230 (2006).

[24] S. Abbasbandy, "Application of He's homotopy perturbation method for Laplace transform", Chaos, Solitons \& Fractals 30, 1206-1212 (2006).

[25] A. Beléndez, A. Hernández, T. Beléndez and A. Márquez, "Application of the homotopy perturbation method to the nonlinear pendulum”, Eur. J. Phys. 28, 93-104 (2007).

[26] A. Beléndez, A. Hernández, T. Beléndez, E. Fernández, M. L. Álvarez and C. Neipp, "Application of He's homotopy perturbation method to the Duffing-harmonic oscillator", Int. J. Non-linear Sci. Numer. Simulation 8 (1), 79-88 (2007).

[27] D. D. Ganji and A. Sadighi, "Application of He's homotopy-perturbation method to nonlinear coupled systems of reaction-diffusion equations", Int. J. Non-linear Sci. Numer. Simulation 7 (4), 411-418 (2006). 
[28] S. Abbasbandy, “A numerical solution of Blasius equation by Adomian's decomposition method and comparison with homotopy perturbation method", Chaos, Solitons \& Fractals 31, 257-260 (2007).

[29] A. Siddiqui, R. Mahmood and Q. Ghori, "Thin film flow of a third grade fluid on moving a belt by He's homotopy perturbation method", Int. J. Non-linear Sci. Numer. Simulation 7 (1), 15-26 (2006).

[30] J. H. He, "Homotopy perturbation method for solving boundary value problems", Phys. Lett. A 350, 87-88 (2006).

[31] M. Rafei and D. D. Ganji, "Explicit solutions of Helmhotlz equation and fifth-order KdV equation using homotopy perturbation method", Int. J. Non-linear Sci. Numer. Simulation 7 (3), 321-328 (2006).

[32] D. D. Ganji, “The application of He's homotopy perturbation method to nonlinear equations arising in heat transfer", Phys. Lett. 355, 337-341 (2006).

[33] M. S. H. Chowdhury and I. Hashim, "Application of homotopy-perturbation method to nonlinear population dynamics models", Phys. Lett. A 368, 251-258 (2007).

[34] P. D. Ariel and T. Hayat, "Homotopy perturbation method and axisymmetric flow over a stretching sheet”, Int. J. Non-linear Sci. Numer. Simulation 7 (4), 399-406 (2006).

[35] M. S. H. Chowdhury and I. Hashim, "Solutions of time-dependent Emden-Fowler type equations by homotopy-perturbation method", Phys. Lett. A 368, 305-313 (2007).

[36] M. S. H. Chowdhury and I. Hashim, "Solutions of a class of singular second-order IVPs by homotopy-perturbation method", Phys. Lett. A 365, 439-447 (2007). 
[37] F. Shakeri and M. Dehghan, "Inverse problem of diffusion by He's homotopy perturbation method", Phys. Scr. 75, 551-556 (2007).

[38] M. Dehghan and F. Shakeri, "Solution of a partial differential equation subject to temperature overspecification by He's homotopy perturbation method", Phys. Scr. 75, 778-787 (2007).

[39] H. M. Liu, "Approximate period of nonlinear oscillators with discontinuities by modified Lindstedt-Poincaré method", Chaos, Solitons \& Fractals 23, 577-579 (2005).

[40] M. Rafei, D. D. Ganji, H. Daniali and H. Pashaei, "The variational iteration method for nonlinear oscillators with discontinuities", J. Sound Vib. 305, 614-620 (2007).

[41] B. S. Wu, W. P. Sun and C. W. Lim, "An analytical approximate technique for a class of strongly non-linear oscillators", Int. J. Non-linear Mech. 41, 766-774 (2006). 